\title{
INCIDENCIA DEL CIBERBULLYING EN ADOLESCENTES DE 11 A 17 AÑOS EN PORTUGAL
}

\section{IMPACT OF CYBERBULLYING IN ADOLESCENTS FROM 11 TO 17 IN PORTUGAL}

\author{
Pedro De-Barros Ventura; pedrodebarros@correo.ugr.es \\ Antonio-Manuel Rodríguez-García; arodrigu@ugr.es \\ Universidad de Granada \\ José-María Sola Reche; jsola@ua.es \\ Universidad de Alicante
}

\section{RESUMEN}

En este artículo se presenta una investigación nacional llevada a cabo en el contexto de Portugal y que ha tenido por objetivo analizar la incidencia del cyberbullying en adolescentes portugueses de entre 11 y 17 años. Para ello, se ha llevado a cabo un estudio cuantitativo empleando un cuestionario ad hoc como instrumento de recogida de información. La muestra ha estado compuesta por 739 estudiantes de 15 distritos del país. Los resultados señalan que un $19,5 \%$ de la muestra ha sido víctima de cyberbullying, el cual se ha manifestado principalmente mediante insultos y amenazas a través de Internet, teléfono e email, así como por la distribución de fotografías de contenido sexual sin consentimiento de las víctimas.

Palabras clave: cyberbullying, TIC, violencia escolar, adolescencia.

\begin{abstract}
In this article we present a national research carried out in the context of Portugal. The aim of this study was to analyze the incidence of cyberbullying in Portuguese adolescents between 11 and 17 years old. For this, a quantitative study has been carried out using an ad hoc questionnaire as an information collection tool. The sample was composed of 739 students from 15 districts of the country. The results indicate that $19.5 \%$ of the sample has been a victim of cyberbullying. The aggression has been manifested mainly through insults and threats through the Internet, telephone and email, as well as by the distribution of sexual photographs without the victims' permission.
\end{abstract}

Keywords: cyberbullying, ICT, school violence, adolescence.

\section{INTRODUCCIÓN.}


Las indagaciones sobre cyberbullying se han incrementado en el panorama internacional de la investigación en ciencias sociales (e.g.: Chester et al., 2015; Conway, Gomez-Garibello, Talwar, y Shariff, 2016; Cross et al., 2016; Ferreira, Simão, Ferreira, Souza, y Francisco, 2016; Meter \& Bauman, 2015; Rice et al., 2015; Sosa y Capafóns, 2015), especialmente tras la irrupción de las Tecnologías de la Información y la Comunicación en todas las esferas de nuestra sociedad (Amado, Matos, Pessoa, y Jäger, 2009; Garaigordobil, 2011; Giménez, Hunter, Durkin, Arnáiz, \& Maquilón, 2015; Lucas, Pérez de Albéniz, y Giménez, 2016).

El cyberbullying, ciberabuso, cibermatonaje o acoso cibernético, se entiende como una variante del bullying tradicional cuyo nacimiento se remonta al surgimiento y auge de las Tecnologías de la Información y la Comunicación. Se concibe como un tipo de agresión que se produce de manera constante y repetida durante un período prolongado haciendo uso de las TIC (principalmente Internet y teléfono móvil) cuyas incidencias manifiestan como resultados una serie de implicaciones negativas severas para la persona, tales como ansiedad, depresión, estrés, pensamientos suicidas, irritabilidad, nerviosismo, miedo, baja autoestima, somatización, sentimientos de indefensión, trastornos del sueño, dificultades de concentración, problemas de rendimiento académico, dificultades para relacionarse con otras personas, entre otras (Garaigordobil, 2011).

Es de tal magnitud el problema, que la Organización Mundial de la Salud (OMS), así como el Fondo de las Naciones Unidas para la Infancia (UNICEF) destacan que el bullying es un problema global que atañe a toda la población y que afecta a uno de cada tres estudiantes de entre 13-15 años de edad en todo el mundo, siendo datos extremos, por ejemplo, en Samoa donde la situación afectaría a tres de cada cuatro escolares (UNICEF, 2014). Un estudio longitudinal llevado a cabo en 33 países de Europa y Norteamérica entre los años 2002 y 2010 llegó a la conclusión de que el bullying es un problema que afecta prácticamente a todos los países analizados, llegando a afectar a un tercio de los jóvenes de entre 11 y 15 años, reafirmando los datos anteriormente dados (Chester et al., 2015). A nivel nacional, el problema cobra especial relevancia más aún cuando somos testigos diariamente de noticias en los distintos medios de comunicación acerca de sucesos de esta índole que acabaron en tragedias inhumanas. Concretamente, hemos sido refrendatarios de suicidios debido a insultos y amenazas reiteradas por parte de compañeros del colegio, ofensas en torno a las preferencias sexuales, entre otros.

Por todos es sabido la importancia que tiene la institución escolar como medio de socialización de los jóvenes, siendo el lugar clave donde comienzan las relaciones con los iguales y con los docentes. Es una etapa de vital importancia ya que a través de ella se va forjando la personalidad del individuo, sus valores y el papel que éste tiene con la sociedad. Las interacciones sociales contribuyen al desarrollo cognitivo, social, afectivo y psicológico de las nuevas generaciones, por lo que se convierten en aspectos decisivos en la construcción de la identidad de la persona. Por su parte, la pre-adolesencia y la adolescencia se conciben como etapas especialmente relevantes y a la vez complicadas en el desarrollo de la persona debido, entre otros factores, a la sucesión de cambios físicos, fisiológicos y psicológicos que ha de enfrentar el sujeto (Caetano, Freire, Simão, Martins, \& Pessoa, 2016). Sin embargo, todas ellas guardan algo en común y se trata de la necesidad de aceptación y de pertenencia a un grupo de referencia.

Hasta hace algunos años, los agentes socializadores se centraban fundamentalmente en la familia, los grupos de pares, la escuela y el trabajo. Sin embargo, desde hace algunas décadas, la penetración de la tecnología dio lugar al reconocimiento de los medios de comunicación 
(televisión, Internet, dispositivos móviles, etc.) como agentes relevantes en la interacción social de los sujetos y, por tanto, como espacio de intercambio de experiencias.

En este escenario se ponen a disposición de las nuevas generaciones una cantidad interminable de posibilidades de interacción con los demás (whatsapp, redes sociales, e-mail, plataformas virtuales, juegos online, blogs, foros, páginas web...) a los que se puede acceder desde diferentes dispositivos portables o fijos. De esta manera, tenemos a nuestro alcance un acceso inmediato a gran cantidad de información, posibilidades de interacción, expresión y, en definitiva, de dejar nuestra huella digital en la red. Sin embargo, cuando se hace un mal uso de las TIC podemos encontrarnos con casos de acoso escolar o cyberbullying, y en el que los medios son utilizados para insultar, difamar, chantajear, coaccionar y/o amenazar a otra persona, especialmente por la facilidad que éstos conceden a los agresores para atacar a los acosados (Álvarez García et al., 2011; Arnáiz Sánchez, Cerezo Ramírez, Giménez, \& Maquilón Sánchez, 2016; Cerezo Ramírez, 2012; Garaigordobil Landazabal, 2014; Sosa Castilla \& Capafóns Bonet, 2015).

Dentro de las diferentes tipologías y manifestaciones del cyberbullying (Kessel Schneider, O'Donnell, \& Smith, 2015; Kowalski, Agatston, \& Limber, 2010; Lucas Molina et al., 2016; Meter \& Bauman, 2015) señalamos las siguientes:

- Distribución de multimedia: subir a la red imágenes o vídeos (reales o fotomontajes) sin el permiso de la víctima y/o donde el objetivo principal sea hacer daño y perjudicar al acosado.

- Suplantación de identidad: registrarse en páginas webs, chats, redes sociales... con el objetivo de atentar contra la integridad de la víctima, ya sea donde se carguen fotos para votar a la persona más fea o creándose perfiles en su nombre y actuando como tal.

- Robo de identidad: hacerse con las claves de los elementos personales de la víctima (móvil, redes sociales, correo electrónico) y actuar en su nombre.

- Difamación: crear rumores y comentarios peyorativos sobre una persona, especialmente cuando estos proceden de la invención de los agresores, con el objetivo de dañar a la persona.

- Insultos: faltas de respeto hacia la persona de manera directa donde se le dicen una serie de palabras que tienen por objeto herir sus sentimientos.

- Amenazas: gestos que tienen por objetivo dañar o extorsionar a la víctima si ésta no accede a realizar las peticiones del agresor.

- Exclusión de juegos online: no dejar participar a la víctima en juegos online, a los que se les dificulta su participación.

- Sexting: consiste en la difusión de imágenes o vídeos con alto contenido erótico y/o sexual con el objetivo de provocar la humillación en el protagonista de los audiovisuales.

- Grooming: un adulto se hace pasar por menor de edad para contactar con jóvenes y ganarse su amistad.

Atendiendo a sus protagonistas encontramos tres roles bien diferenciados: el atacador, la víctima y el observador (Hollá, 2016; Kowalski et al., 2010). Más específicamente, investigaciones llevadas al efecto señalan e identifican los tipos de roles que caracterizan a los sujetos que han experimentado un proceso de intimidación o acoso manifestado a través de bullying: por un lado, la víctima que sirve como "cabeza de turco" para un grupo, que carece de habilidades y en algunas ocasiones se caracteriza por tener una personalidad retraída, 
tímida e introvertida; por otro, la víctima "provocadora", que promueve una reacción adversa en los demás y carece de habilidades para enfrentarse a las situaciones que devienen; $y$, por último, la víctima "agresora", que sería aquella persona que tiende a reproducir las conductas agresivas que ha padecido. Por otro lado, estaría el agresor que sería la persona encargada de atacar a los más débiles y, en último lugar, el observador, que sería la persona testigo de la agresión pero que ni ataca ni es atacado por el agresor ni la víctima (Garaigordobil, 2014; Lucas Molina et al., 2016).

Para finalizar, atendiendo a la literatura científica los efectos académicos más notorios del bullying y cyberbullying en los alumnos acosados pueden incluir: desinterés, falta de motivación escolar y académica, problemas de concentración, de aprendizaje, dificultades en el rendimiento académico, absentismo escolar o incluso abandono de la institución (Garaigordobil, 2011; Kowalski et al., 2013; Kowalski, Giumetti, Schroeder, \& Lattanner, 2014). Por ende, si los efectos del bullying y cyberbullying son devastadores para cualquier persona, tanto en lo psicológico como en lo social, los resultados académicos pueden ser un indicio importante para que los docentes se hagan eco de que algo no está yendo del todo bien.

\section{METODOLOGÍA}

La presente investigación forma parte de otra de mayor envergadura llevada a cabo en el contexto de Portugal durante el curso escolar 2016-2017 y tiene como objetivo conocer e identificar la incidencia del cyberbullying en y adolescentes de entre 11 y 17 años, pertenecientes al tercer ciclo de enseñanza básica de Portugal. Para ello se ha llevado a cabo un estudio de corte metodológico cuantitativo a través de un diseño no experimental y usando un cuestionario ad hoc como técnica de recogida de información. A continuación, detallamos de manera pormenorizada la muestra, instrumentos y procedimiento seguido.

\section{MUESTRA PARTICIPANTE}

En total participaron 934 estudiantes, siendo 191 los alumnos que formaron parte del estudio piloto y 739 alumnos quienes finalmente fueron seleccionados para participar en la investigación. Para la selección de la misma seguimos un muestreo probabilístico aleatorio estratificado (Hernández, Fernández y Baptista, 2006), con el objetivo de asegurar la representación de cada grupo de edad en la muestra. Para garantizar este tipo de muestreo se procedió a enviar los cuestionarios a los centros escolares de las 11 distritos de Portugal, recibiendo respuesta de 739 estudiantes (79,1\%), que serán quienes conformen nuestra muestra debido a la mayor objetividad y rigurosidad en el proceso de indagación (Tabla 1).

\begin{tabular}{|l|l|l|}
\hline Distrito & Cuantía & Porcentaje \\
\hline Açore & 25 & $3,38 \%$ \\
\hline Aveiro & 159 & $21,52 \%$ \\
\hline Beja & 23 & $3,14 \%$ \\
\hline Braga & 2 & $0,27 \%$ \\
\hline Castelo Branco & 29 & $3,95 \%$ \\
\hline Évora & 12 & $1,62 \%$ \\
\hline Faro & 42 & $5,68 \%$ \\
\hline
\end{tabular}




\begin{tabular}{|l|l|l|}
\hline Guarda & 1 & $0,14 \%$ \\
\hline Leiria & 2 & $0,27 \%$ \\
\hline Lisboa & 68 & $9,2 \%$ \\
\hline Oporto & 132 & $17,86 \%$ \\
\hline Santarém & 43 & $5,82 \%$ \\
\hline Setúbal & 80 & $10,83 \%$ \\
\hline Viana do Castelo & 119 & $16,10 \%$ \\
\hline Viseu & 2 & $0,27 \%$ \\
\hline Total & 739 & $100 \%$ \\
\hline
\end{tabular}

Tabla 1. Distribución de la muestra por distrito de Portugal

Como podemos observar en las tablas siguientes (sexo, edad y curso de los participantes), cabe destacar que la mayoría de las participantes pertenecen al sexo femenino y, en menor medida, al sexo masculino (Tabla 2). Contamos con participantes de diferentes edades comprendidas entre los 11 y los 17 años (Tabla 3). Por curso, tenemos una representación equitativa de los tres analizados (Tabla 4). Si bien es cierto que el 70,9\% de la muestra lo componen alumnos que cursan $7^{\circ}$ y $8^{\circ}$, cuyas edades oscilan entre los 12 y los 14 años, y el $29,1 \%$ restante alumnos de $9^{\circ}$ curso, con edades entre 15 y 17 años, destacando un porcentaje de repetidores en este último curso. En definitiva, el rango de representación de edad se enmarca dentro del esperado para el objetivo de investigación en el tercer ciclo de la educación básica de Portugal (12-15 años).

\begin{tabular}{|l|l|}
\hline Sexo & Porcentaje \\
\hline Mujer & $53,7 \%$ \\
\hline Hombre & $46,3 \%$ \\
\hline
\end{tabular}

Tabla 2. Sexo de la muestra

\begin{tabular}{|l|l|}
\hline Edad & Porcentaje \\
\hline 11 & $0,5 \%$ \\
\hline 12 & $16,5 \%$ \\
\hline 13 & $28,6 \%$ \\
\hline 14 & $29,2 \%$ \\
\hline 16 & $15,6 \%$ \\
\hline 17 & $6,8 \%$ \\
\hline
\end{tabular}

Tabla 3. Edad de la muestra

\begin{tabular}{|l|l|}
\hline Curso & Porcentaje \\
\hline $7^{\circ}$ & $36,3 \%$ \\
\hline $8^{\circ}$ & $34,6 \%$ \\
\hline $9^{\circ}$ & $29,1 \%$ \\
\hline
\end{tabular}

Tabla 4. Curso de la muestra

\section{INSTRUMENTOS DE RECOGIDA DE INFORMACIÓN}

Con el objetivo de valorar la incidencia del cyberbullying entre los alumnos del tercer ciclo de enseñanza básica en Portugal se construyó un instrumento ad hoc para tal efecto (De 
Barros, 2010). Los ítems estuvieron compuestos por diferentes tipologías de respuestas: respuesta directa, casilla de verificación, de respuesta múltiple con opción no vinculante y con la opción de añadir otra respuesta, permitiendo ir más allá de los ítems señalados. En total, el cuestionario consta de 67 ítems.

El cuestionario se diseñó en torno a cinco áreas temáticas: características de la muestra 10 ítems- (sexo, clase, edad, repetidor, idioma, ubicación geográfica, constitución física, apariencia física y tipo de estudiante en comparación con las calificaciones obtenidas por los compañeros), víctimas de bullying y cyberbullying -23 ítems- (agresión, tipos de agresión, consecuencias y acciones posteriores), agresores de bullying y cyberbullying -21 ítems- (agresión, tipos de agresión, motivación, acciones después de a agresión), observadores en el cyberbullying - 5 ítems- (medios de agresión, tipos de agresión, consecuencias y acciones después de la agresión) y, finalmente, uso de las Tecnologías de la Información y la Comunicación -7 ítems-.

Concretamente, la investigación que aquí presentamos pertenece al cuerpo de víctimas de bullying y cyberbullying, cuyos 23 ítems estuvieron comprendidos en una Escala Likert de 1 a 3 (1:Nunca, 2:Algunas veces, 3:Frecuentemente). En este momento es preciso señalar que las preguntas se realizaron de manera indirecta para que la víctima no se sintiese condicionada al responder. A su vez, el cuestionario fue diseñado para que se pudiera determinar la incidencia de la agresión no por una posición positiva hacia la pregunta de manera singular, sino a través de la suma de las cuestiones relacionadas con el tipo de agresión (cyberbullying).

El instrumento fue validado mediante análisis de expertos y a través del cálculo del alfa de Cronbach, obteniendo un valor de 0,876. A su vez, el cuestionario fue validado por la Dirección General de Innovación y Desarrollo Curricular (DGIDC), del Ministerio de Educación de Portugal, aprobado con número 0090600001.

\section{PROCEDIMIENTO}

El procedimiento que seguimos fue, en primer lugar, informar a todos los centros escolares del país con niveles de tercer ciclo de enseñanza básica sobre la relevancia del estudio y la importancia de participación el mismo. De este modo, se envió un e-mail para solicitar la participación de los centros y de los alumnos en la investigación. Aunque no se recibió respuesta de todos, se contó con la presencia de la mayoría de los distritos del país y un total de 33 centros educativos. El cuestionario se probó durante un cierto período de tiempo a través de su puesta online y solicitando a la gente inmediata su colaboración en la misma (respuestas obtenidas fuera del centro escolar -195 sujetos-). Posterior a ella se realizaron una serie de correcciones que dieron lugar a la validación por el Ministerio de Educación, siendo subsiguientemente enviado a los respectivos centros escolares.

El instrumento enviado a los centros llevaba consigo una lista de procedimientos que debían seguirse para cumplimentar los cuestionarios, así como una solicitud de autorización a los padres que permitiesen obtener las respuestas de sus hijos de una manera ética y responsable. Del mismo modo, los instrumentos iban acompañados de una serie de claves para permitir identificar la fecha, hora, año escolar, distrito y evitar duplicidad de resultados procedentes de rellenarlos en casa o en el centro.

Una vez cumplimentado por los estudiantes, se recabaron los datos a través del paquete estadístico SPSS Statistics (v.23), realizándose diversos análisis descriptivos que tuviesen por objetivo contextualizar a la muestra participante según las características que anteriormente 
hemos señalado, así como correlaciones bivariadas que nos permitieron establecer relaciones entre las distintas variables analizadas.

El análisis del grupo de víctimas de cyberbullying se llevó a cabo de manera concienciada para cotejar los resultados procedentes de la suma de las respuestas, de manera que se tomaron aquellos datos procedentes de las personas que manifestaban ser víctimas de cyberbullying algunas veces o de manera frecuente en, al menos, tres ocasiones (ítems relacionados).

\section{RESULTADOS}

La estructura de los resultados que presentamos se dividen en diferentes dimensiones bajo la óptica de las víctimas: en primer lugar, encontramos el tipo de agresión realizada a través de la red en función del sexo y la frecuencia recibida; en segundo lugar, el tipo de agresión en función de la edad de los alumnos; en tercer lugar, la identificación del agresor de cyberbullying; en cuarto lugar, las razones por las que se piensa que el agresor realiza cyberbullying; y, en último lugar, un panorama general del impacto del cyberbullying por distrito y como problema nacional.

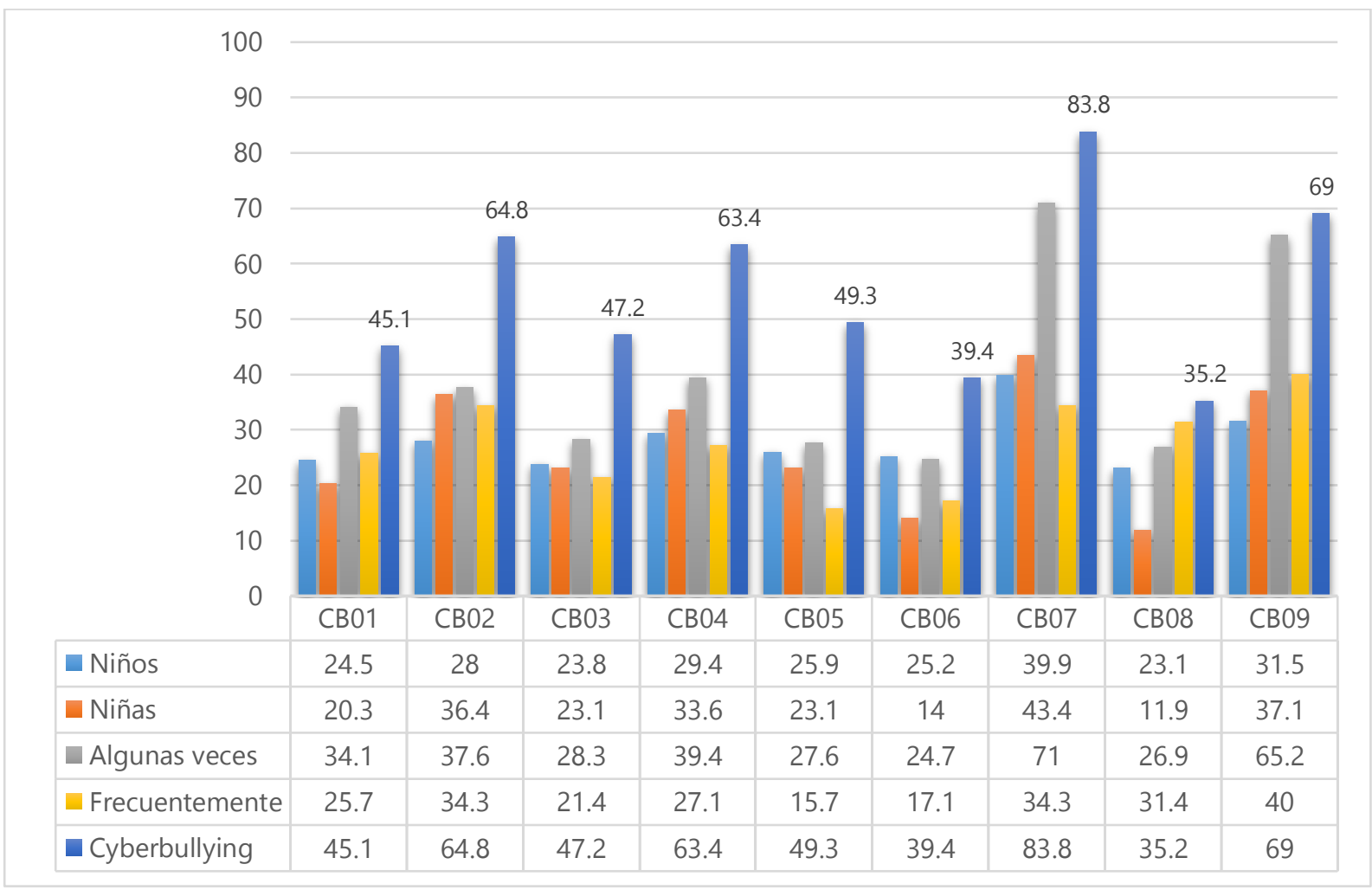

Figura 1. Tipos de agresión en función del sexo y la frecuencia recibida. Elaboración propia.

Atendiendo a los resultados expresados en la Figura 1, donde las siglas se corresponderían con: CB01: Suplantación y robo de identidad, CB02: Amenazas vía mensaje o e-mail; CB03: Difusión de información personal sin consentimiento; CB04: Insultos repetitivos vía mensaje o email; CB05: Difamación; CB06: Publicación de contenido audiovisual personal sin permiso; CB07: Ofensas vía Internet o teléfono; CB08: Exclusión de juegos o grupos online; y CB09: Distribución de fotos íntimas, encontramos unos datos especialmente significativos. En primer lugar, los resultados nos muestran una diferencia en torno al sexo de los alumnos siendo las víctimas masculinas quienes mayor experimentan suplantación de su identidad en sus cuentas 
personales o robo de contraseñas (24,5\%); difusión de información personal sin permiso (23,8\%), aunque no muy significativo en comparación con las mujeres; difamación (25,9\%); publicación de vídeos o fotos sin permiso del que es grabado o fotografiado, tales como peleas o reyertas filmadas y difundidas posteriormente $(25,2 \%)$; y exclusión de juegos o grupos interactivos online $(23,1 \%)$. Por su parte, las chicas son un foco mayor de agresiones del tipo amenazas a través de mensajes, chats o e-mail (36,4\%); insultos reiterados a través de medios electrónicos (33,6\%); ofensas a través de la red o del teléfono móvil $(43,4 \%)$; y en lo que respecta a la solicitud de fotos íntimas y personales (37,1\%).

Por otro lado, si analizamos las agresiones emitidas a través de las TIC en función del grado de ocurrencia en las víctimas, encontramos que los ataques recibidos con mayor frecuencia serían: la distribución de fotografías de carácter sexual (sexting) con un 40\%, seguido de amenazas a través de mensajes o e-mails u ofensas a través de la red, ambas con un 34,3\%. De las personas que afirman recibir en algunas ocasiones cyberbullying a través de las TIC, encontramos que las prácticas más frecuentadas son: humillaciones y ofensas a través de Internet o el móvil (71\%), sexting $(65,2 \%)$ e insultos constantes a través de mensajes o correo electrónico $(39,4 \%)$.

A nivel general, encontramos que las agresiones más comúnmente empleadas por los acosadores a través de las TIC son: ofensas a través de Internet o móvil (83,8\%), distribución de fotos íntimas o sexting (69\%), amenazas a través de los medios electrónicos (64,8\%) e insultos reiterativos a través de los mismos medios $(63,4 \%)$.

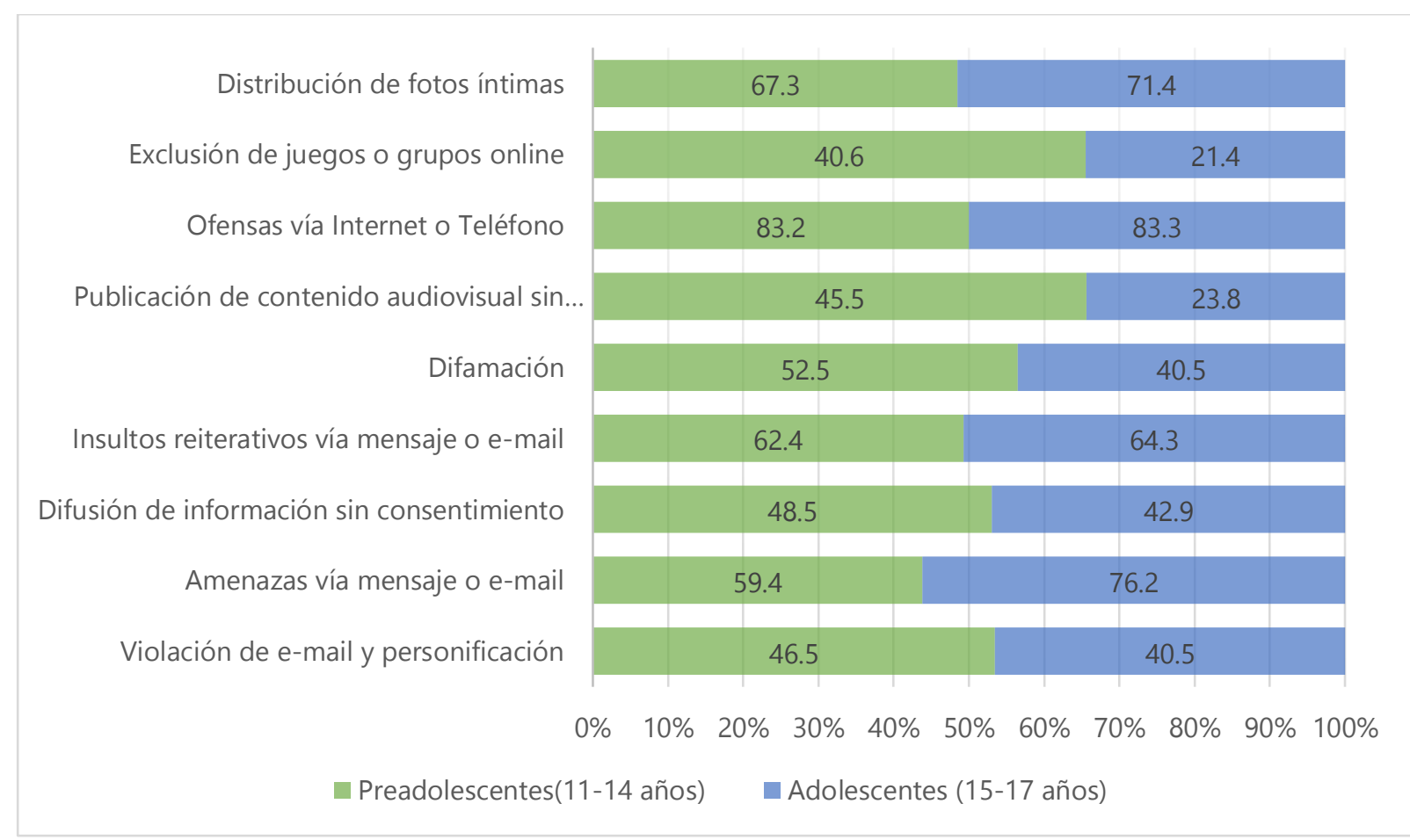

Figura 2. Tipos de agresión en función de la edad.

A pesar de que la literatura científica no establece un acuerdo para diferenciar la adolescencia de la preadolescencia, encontramos una serie de convergencias entre distintos autores, de forma que tomamos dichos datos como referencia para el corte de dos grupos homogéneos en función de la edad (preadolescencia 11-14 años y adolescencia 15-17 años) y el tipo de agresión recibida. De este modo, los resultados evidencian una mayor apariencia de agresiones relacionadas con la ofensiva a través de medios electrónicos $(83,2 \%)$, distribución de fotos 
íntimas (67,3\%), insultos a través de e-mail o mensajes $(62,4 \%)$ y amenazas a través de los mismos medios (62,4\%) en estudiantes de entre 11 y 14 años.

En lo que respecta a las víctimas de entre 15 y 17 años, encontramos una mayor frecuencia de ofensivas a través de dispositivos electrónicos (83,3\%), amenazas (76,2\%), distribución de fotos íntimas $(71,4 \%)$ y difusión de información sin consentimiento (42,9\%). En último lugar se encontrarían las agresiones relacionadas con la publicación de contenido audiovisual sin consentimiento $(23,8 \%)$ exclusión de juegos o grupos online $(21,4 \%)$.

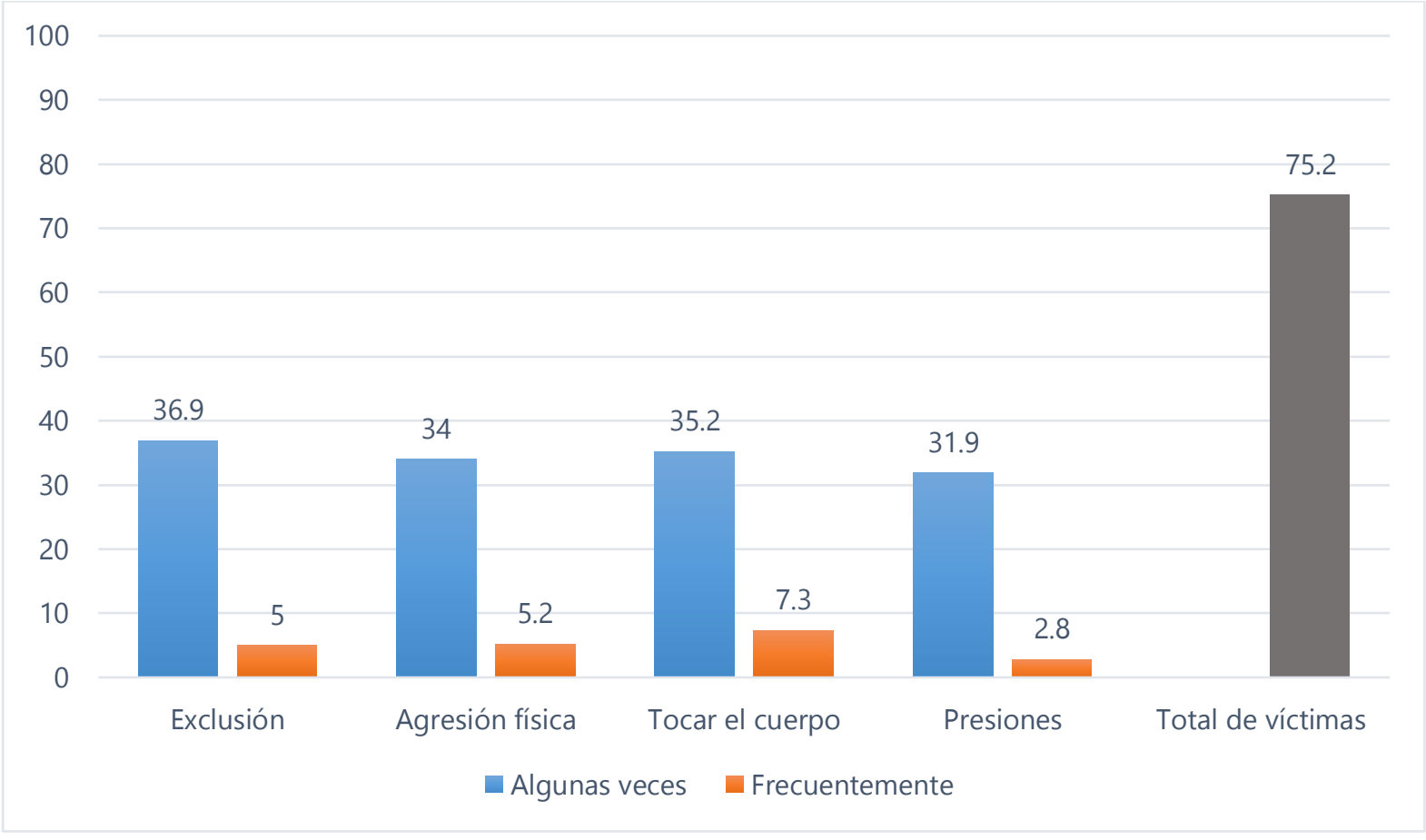

Figura 3. Bullying entre las víctimas de cyberbullying.

En la figura 3 observamos que existen un porcentaje bastante significativo de víctimas de cyberbullying que, a su vez, experimentan episodios de bullying (75,2\%). Datos muy reveladores ya que, por un lado, evidencian que la mayoría de víctimas de cyberbullying también padecen agresiones e intimidaciones de manera presencial, y solamente el $24,8 \%$ restante lo sufre a través de las TIC. Este tipo de agresiones vienen caracterizadas por la exclusión $(36,9 \%)$, tocamientos del cuerpo e invasión de la intimidad (35,2\%), agresiones físicas (34\%) y presiones (31,9\%). Para aquellos que lo sufren con una frecuencia mayor encontraríamos: invasión del cuerpo (7,3\%), agresión física (5,2\%), exclusión (5\%) y presiones $(2,8 \%)$. 


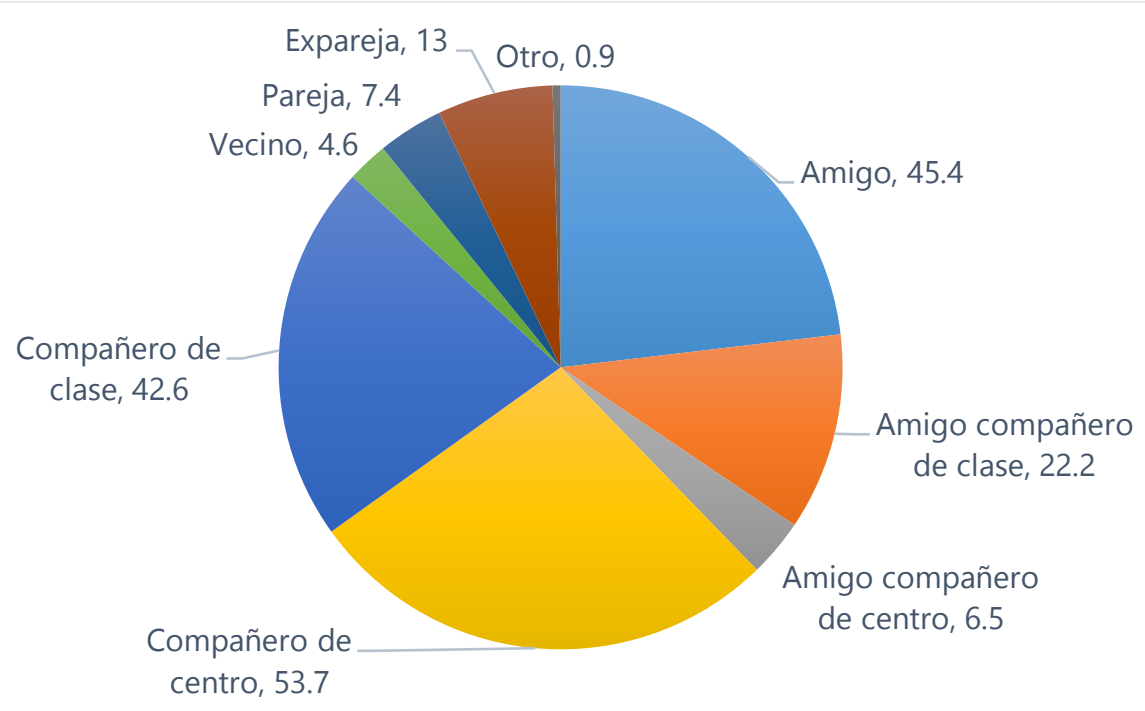

Figura 4. Identificación del agresor.

A diferencia del bullying, el cyberbullying es una práctica que se puede cometer ocultando la identidad del agresor. Sin embargo, las víctimas encuestadas señalaron un porcentaje muy alto de identificación del agresor $(76,1 \%)$, porcentaje muy significativo y similar al número de víctimas que padecen bullying y cyberbullying al mismo tiempo. Según las víctimas, los agresores son más frecuentemente compañeros del centro donde estudian (53,7\%), amigos $(45,4 \%)$, compañeros de la propia clase $(42,6 \%)$ y amigos compañeros de clase $(22,2 \%)$. En menor medida encontramos vecinos $(4,6 \%)$, amigos compañeros del centro $(6,5 \%)$, parejas $(7,4 \%)$ y exparejas (13\%).

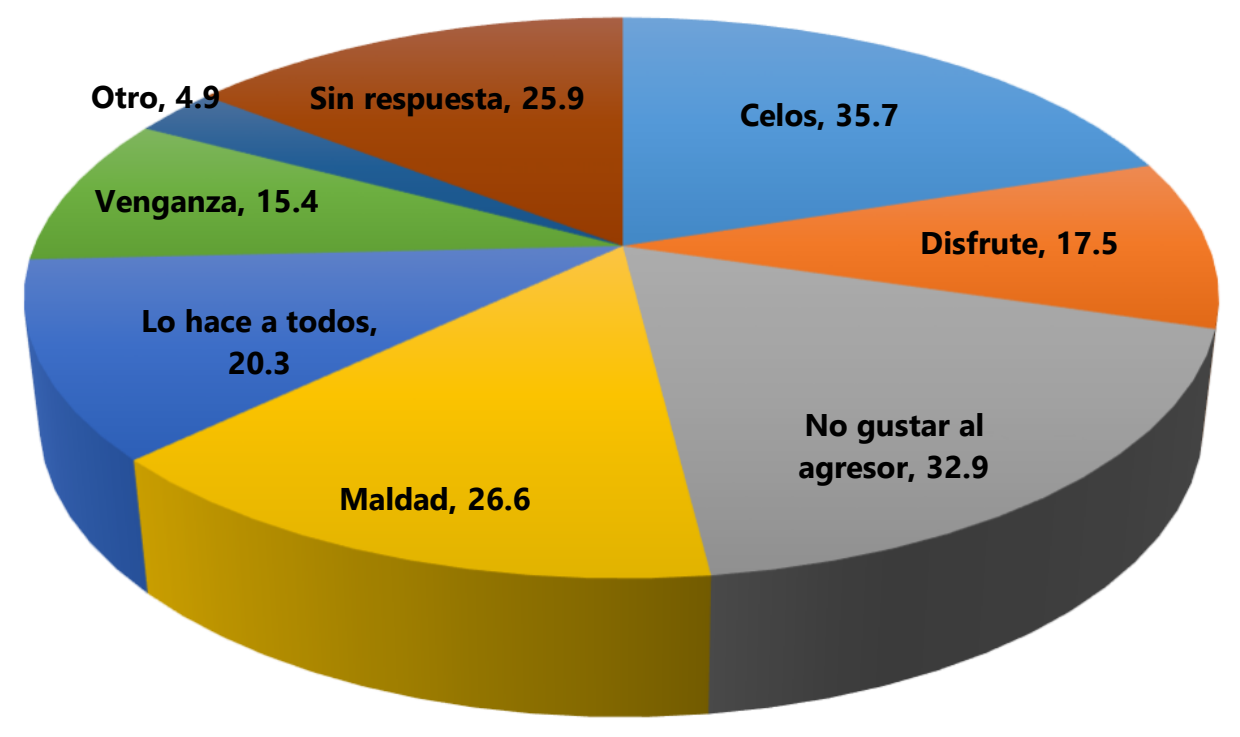

Figura 5. Razones por las que el agresor realiza cyberbullying. 
Por otro lado, al preguntar a las víctimas las motivaciones que llevan a los agresores a emitir acoso a través de las TIC encontramos una serie de razones que señalan como más comunes: celos por cuestiones académicas o personales (35,7\%), no ser del gusto o agrado de la persona que agrede (32,9\%) y la maldad de los atacantes (26,6\%). A su vez, llama la atención que un $25,9 \%$ no encuentra respuesta alguna que pueda explicar los sucesos de intimidación.

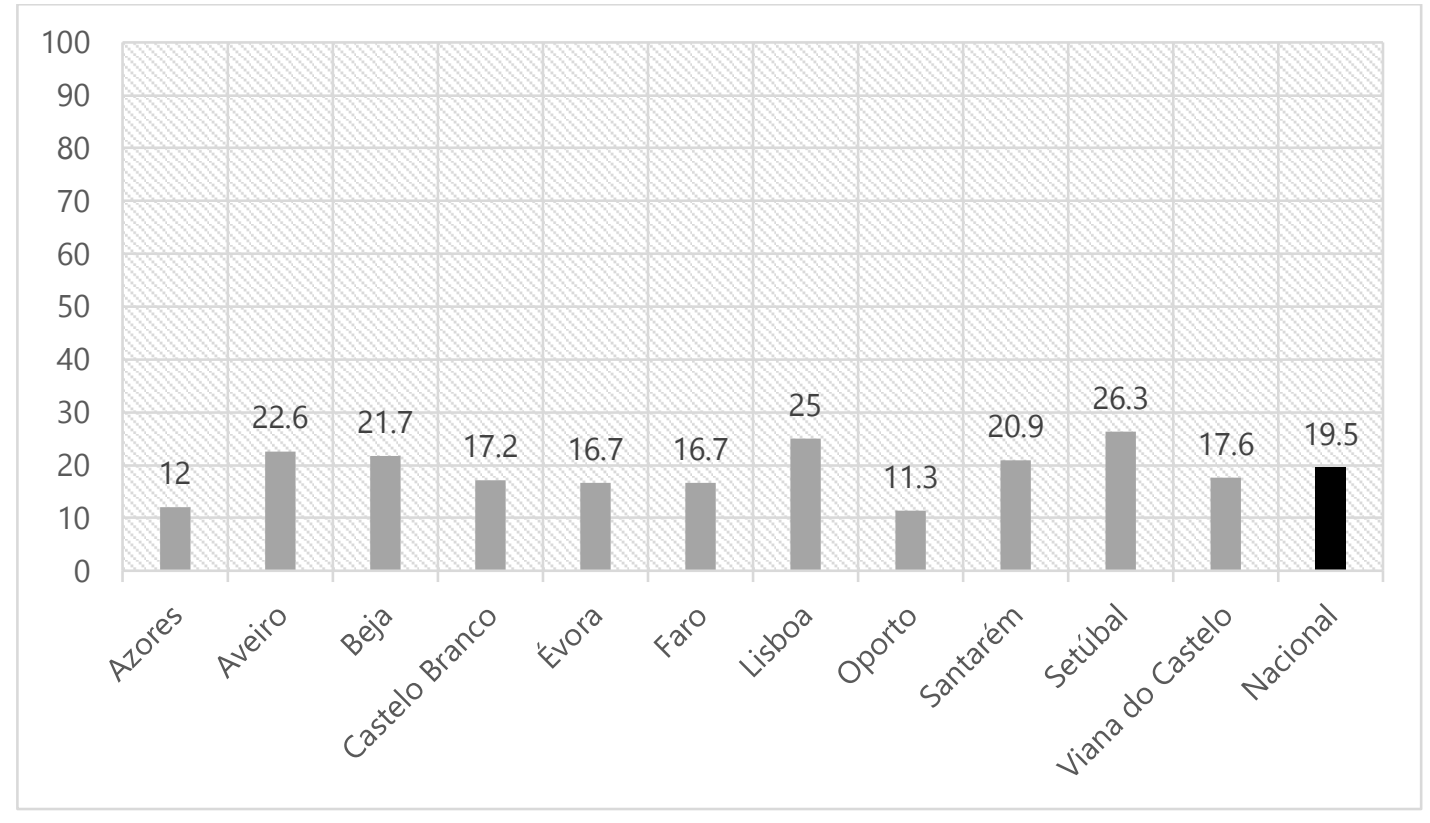

Figura 6. Impacto del cyberbullying por distrito y nación.

Finalmente, encontramos que el problema del cyberbullying afecta a un $19,5 \%$ de los estudiantes en el panorama nacional de Portugal, cifra muy alarmante que interfiere en el correcto desarrollo de los estudiantes del tercer ciclo de enseñanza básica en este país. Si analizamos más concretamente por distritos, Setúbal (26,3\%), Lisboa (25\%) y Aveiro (22,6\%) son las regiones más afectadas por el problema. En el lado opuesto encontraríamos a Oporto $(11,3 \%)$ y las Azores (12\%). Sin embargo, en mayor o menor porcentaje, existe un acoso manifiesto a través de las TIC en todas las regiones que se ofertaron para cumplimentar la investigación.

\section{DISCUSIÓN Y CONCLUSIONES}

La presente investigación ha tenido por objetivo conocer e identificar la incidencia del cyberbullying en escolares preadolescentes y adolescentes de entre 11 y 17 años, pertenecientes al tercer ciclo de enseñanza básica de Portugal. A grandes rasgos, podemos inferir que la prevalencia media nacional del cyberbullying en el grupo de estudiantes que han actuado como foco de la muestra se sitúa en un 19,5\%, constituyéndose como un problema nacional que afecta de manera notoria al contexto educativo portugués. Investigaciones similares llevadas al efecto en el mismo contexto obtienen resultados similares, tales como la Gaspar et al. (2012), con casi el $16 \%$ de la población. A su vez, un estudio a gran escala llevado a cabo internacionalmente encontraron que los alumnos portugueses de 11 a 15 años eran víctimas de esta práctica en torno al 11,5\% y el 16\%, mientras que los agresores se encontraban en torno al 7,5\% y el 11,5\% (Currie et al., 2012).

Sin embargo, difieren de otras investigaciones que encuentran un menor porcentaje de incidencia del cyberbullying, tales como Coelho, Sousa, Marchante, Bras, \& Romao (2016), quienes encontraron un porcentaje menor en el grupo de víctimas (10,1\%) aunque 
solamente llevado a cabo en Lisboa. En el mismo contexto y con una muestra del tercer ciclo de enseñanza básica, la investigación de Freire, Simão, \& Ferreira (2006) encuentra un $7 \%$ de participación de los estudiantes en conductas de bullying, (4,5\% son víctimas y $2,5 \%$ agresores).

A nivel internacional, Arnáiz Sánchez, Cerezo Ramírez, Giménez, \& Maquilón Sánchez (2016) encontraron un impacto del 7,7\% en jóvenes de entre 12 y 21 años en estudiantes españoles, aunque Álvarez-García et al. (2012) señalaban que el 35,4\% de alumnos asturianos habían padecido episodios de cyberbullying. En Eslovaquia se encontró que el $42,7 \%$ con una población de alumnos de 11 a 18 años que el 42,7\% habían sido víctimas de cyberbullying (Hollá, 2016). En Irlanda se encontró un porcentaje mínimo de cyberbullying entre alumnos de 9 y 13 años $(3,4 \%)$ según los estudios de Purdy \& York (2016). En Estados Unidos, Conway et al. (2016) encontraron que el problema afecta al $45 \%$ de estudiantes de entre 8 y 16 años de edad de escuelas públicas en Toronto y Montreal. Como observamos, cada contexto parte de unas circunstancias peculiares y específicas, por lo que no existe un acuerdo entre las investigaciones, obteniendo algunas de ellas mayores porcentajes de acosados que otras. No obstante, tal y como señaló el informe internacional de UNICEF (2014), el cyerbullying es un problema internacional que intercede y padece un tercio de la población de escolares entre 13 y 15 años.

En relación al sexo no se han encontrado diferencias especialmente significativas sobre quiénes toleran el papel de la víctima de manera más frecuente en el bullying cibernético, hecho que avalan las investigaciones de Álvarez-García et al., (2011), Coelho, Sousa, Marchante, Bras, \& Romao (2016). Sin embargo, las chicas son más propensas a ser agredidas, ofendidas y/o amenazadas a través de llamadas, mensajes o emails. A su vez, también son quienes han experimentado mayores episodios de sexting. Por el contrario, los chicos son quienes padecen más robos y suplantaciones de identidad, difamaciones, distribución de audiovisuales sin consentimiento producto de situaciones conflictivas (abusos, peleas, ...), así como la exclusión de juegos y grupos online (Purdy \& York, 2016).

En referencia a los grupos de edad encontramos diferencias significativas que nos aportan datos acerca de las agresiones recibidas más frecuentemente según la edad de la víctima. De este modo, encontraríamos más agresiones relacionadas con la publicación de audiovisuales sin consentimiento, la exclusión de juegos o grupos online y la difmación en el grupo de escolares de entre 11 y 14 años. Los estudiantes de entre 15 y 17 años son víctimas más propensas a sufrir más comúnmente ofensivas, amenazas e insultos a través del móvil o Internet y peticiones o distribución de fotografías de la sexualidad del alumno(Conway et al., 2016).

Independientemente de la edad y el sexo, los modos de agresión empleados a través de las TIC han sido diversos y con valores porcentuales distribuidos entre sí. Sin embargo, encontramos patrones comunes entre las "técnicas" empleadas por los agresores hacia las víctimas, siendo las que se producen en mayor medida la ofensa a través de Internet y el móvil, la distribución de vídeos e imágenes sobre la intimidad de los acosados (sexting), así como la emisión de amenezas e insultos a través de dispositivos y medios electrónicos (Coelho et al., 2016; Garaigordobil, 2011; Pereira \& Matos, 2015; Purdy \& York, 2016; Smith et al., 2014). Destacan, a su vez, la difamación, la suplantación de la identidad o robo de cuentas y la difusión de información personal sin consentimiento del protagonista. En último lugar se encuentran las prácticas que difunden audiovisuales sin consentimiento del grabado o fotografiado y la exclusión de juegos y grupos online. 
En definitiva, el cyberbullying es un acto de agresión que se canaliza a través de las Tecnologías de la Información y la Comunicación, que le sirven como el medio ideal para llevar a cabo tales prácticas de intimidación. En el caso que nos ocupa, el cyberbullying se ha convertido en un problema global tras la irrupción tecnológica. Diversos factores han contribuido para ello: facilidad de ocultar la identidad, estar en contacto con la víctima, practicar violaciones o agresiones de la intimidad (Kowalski et al., 2014). Tomando como referencia los datos obtenidos en esta investigación, las TIC se han convertido en un medio adicional para extender el acoso escolar a otros medios y esferas, pues los datos obtenidos en este estudio ponen de manifiesto que el $75 \%$ de los alumnos que son víctimas de acoso cibernético también lo son en el contexto escolar, hecho que avalan las investigaciones de Andrade (2012), Coelho et al. (2016), Kessel Schneider et al. (2015), Kowalski et al. (2010, 2014)

\section{Referencias}

Álvarez-García, D., Núñez Pérez, J. C., Pérez, L. Á., González, A. D., Rodríguez Pérez, C., \& González-Castro, P. (n.d.). Violencia a través de las tecnologías de la información y la comunicación en estudiantes de secundaria, 27(1), 221-231. Recuperado de http://revistas.um.es/analesps

Álvarez García, D., Núñez Pérez, J. C., Álvarez Pérez, L., Dobarro, A., Rodríguez Pérez, C., \& González Castro, P. (2011). Violencia a través de las tecnologías de la información y la comunicación en estudiantes de secundaria. Anales de Psicología, 27(1), 221-230.

Amado, J., Matos, A., Pessoa, T., \& Jäger, T. (2009). Cyberbullying: um desafio à investigação e à formação, 13, 301-326. Recuperado de http://www.eses.pt/interaccoes

Andrade, L. C. F. (2012). Bullying e cyberbullying: um estudo num contexto escolar particular cooperativo. Recuperado de https://digituma.uma.pt/bitstream/10400.13/422/1/MestradoLuisaAndrade.pdf

Arnáiz Sánchez, P., Cerezo Ramírez, F., Giménez, A. M., \& Maquilón Sánchez, J. J. (2016). Conductas de ciberadicción y experiencias de \&quot;cyberbullying\&quot; entre adolescentes. Anales de Psicología32(3), 761-769.

Caetano, A. P., Freire, I., Simão, A. M. V., Martins, M. J. D., \& Pessoa, M. T. (2016). Emoções no cyberbullying: um estudo com adolescentes portugueses. Educação E Pesquisa, 42(1), 199212. Recuperado de: https://doi.org/10.1590/S1517-9702201603138125

Cerezo Ramírez, F. (2012). Bullying a través de las TIC. Boletín Científico Sapiens Research, 2(2), 24-29.

Chester, K. L., Callaghan, M., Cosma, A., Donnelly, P., Craig, W., Walsh, S., \& Molcho, M. (2015). Cross-national time trends in bullying victimization in 33 countries among children aged 11, 13 and 15 from 2002 to 2010. The European Journal of Public Health, 25(suppl 2).

Coelho, V. A., Sousa, V., Marchante, M., Bras, P., \& Romao, A. M. (2016). Bullying and cyberbullying in Portugal: Validation of a questionnaire and analysis of prevalence. School Psychology International, 37(3), 223-239. Recuperado de: 


\section{https://doi.org/10.1177/0143034315626609}

Conway, L., Gomez-Garibello, C., Talwar, V., \& Shariff, S. (2016). Face-to-Face and Online: An Investigation of Children's and Adolescents' Bullying Behavior Through the Lens of Moral Emotions and Judgments. Journal of School Violence, 15(4), 503-522. Recuperado de: https://doi.org/10.1080/15388220.2015.1112805

Cross, D., Shaw, T., Hadwen, K., Cardoso, P., Slee, P., Roberts, C., ... Barnes, A. (2016). Longitudinal impact of the Cyber Friendly Schools program on adolescents' cyberbullying behavior. Aggressive Behavior, 42(2), 166-180. Recuperado de: https://doi.org/10.1002/ab.21609

Currie, C., Zanotti, C., Morgan, A., Currie, D., de Looze, M., et al. (Eds). (2012). Social determinants of health and well-being among young people. Health Behaviour in School-aged Children (HBSC) study: International report from the 2009/2010 survey. Health Policy for Children and Adolescents. No. 6. Copenhagen: WHO Regional Office for Europe.

De Barros, P. M. (2010). Incidência e impacto do cyberbullying nos alunos do terceiro ciclo do ensino básico público português (Tesis doctoral). Universidad de Granada: Deparamento de Didáctica y Organización Escolar.

Ferreira, P. C., Simão, A. M. V., Ferreira, A., Souza, S., \& Francisco, S. (2016). Student bystander behavior and cultural issues in cyberbullying: When actions speak louder than words. Computers in Human Behavior, 60, 301-311. Recuperado de: https://doi.org/10.1016/j.chb.2016.02.059

Freire, I. P., Simão, A. M. V., \& Ferreira, A. S. (2006). O estudo da violência entre pares no $3^{\circ}$ ciclodo ensino básico - um questionário aferidopara a população escolar portuguesa. Revista Portuguesa de Educação, 19(2), 157-183.

Hernández, R., Fernández, C., \& Baptista, P. (2006). Metodología de la investigación. México: McGraw-Hill.

Garaigordobil, M. (2011). Prevalencia y consecuencias del cyberbullying: una revisión. Internatlonal Journal of Psychology and Psychologlcal theraPy, 11(2), 233-254.

Garaigordobil Landazabal, M. (2014). Cyberbullying: una nueva forma de violencia entre iguales a través de los medios electrónicos. Revista Padres $Y$ Maestros / Journal of Parents and Teachers, (357), 34-40.

Gaspar, M., Simões, C., Tomé, G., Camacho, I., Ferreira, M., Ramiro, L., Reis, M., Gaspar, T., Veloso, S., Loureiro, N., Borges, A., Alves, J. \& Equipa Aventura Social (2012). A saúde dos adolescentes portugueses.Relatório do estudo HBSC 2010. Recuperado de https://goo.gl/Kc0Aep

Giménez Gualdo, A. M., Hunter, S. C., Durkin, K., Arnaiz, P., \& Maquilón, J. J. (2015). The emotional impact of cyberbullying: Differences in perceptions and experiences as a function of role. Computers \& Education, 82, 228-235. Recuperado de: https://doi.org/10.1016/j.compedu.2014.11.013 
Hollá, K. (2016). Cyberbullying as a negative result of cyber-culture of Slovak children and adolescents: selected research findings. Journal of Language and Cultural Education, 4(2). Recuperado de: https://doi.org/10.1515/jolace-2016-0015

Kessel Schneider, S., O’Donnell, L., \& Smith, E. (2015). Trends in Cyberbullying and School Bullying Victimization in a Regional Census of High School Students, 2006-2012. Journal of School Health, 85(9), 611-620. Recuperado de: https://doi.org/10.1111/josh.12290

Kowalski, R. M., Agatston, P., \& Limber, S. (2010). Cyber bullying : el acoso escolar en la era digital. Desclée de Brouwer.

Kowalski, R. M., Giumetti, G. W., Schroeder, A. N., \& Lattanner, M. R. (2014). Bullying in the digital age: A critical review and meta-analysis of cyberbullying research among youth. Psychological Bulletin, 140(4), 1073-1137. Recuperado de: https://doi.org/10.1037/a0035618

Kowalski, R. M., Limber, S. P., Nishina, A., Juvonen, J., Olweus, D., Hinduja, S., ... al., et. (2013). Psychological, physical, and academic correlates of cyberbullying and traditional bullying. The Journal of Adolescent Health: Official Publication of the Society for Adolescent Medicine, 53(1 Suppl), S13-20. Recuperado de: https://doi.org/10.1016/j.jadohealth.2012.09.018

Lucas Molina, B., Pérez de Albéniz Iturriaga, A., \& Giménez Dasí, M. (2016). La evaluación del \&quot;cyberbullying\&quot;: situación actual y retos futuros. Papeles Del Psicólogo, 37(1), 27-35.

Meter, D. J., \& Bauman, S. (2015). When Sharing Is a Bad Idea: The Effects of Online Social Network Engagement and Sharing Passwords with Friends on Cyberbullying Involvement. Cyberpsychology, Behavior, and Social Networking, 18(8), 437-442. Recuperado de: https://doi.org/10.1089/cyber.2015.0081

Pereira, F., \& Matos, M. (2015). Cyberstalking entre adolescentes: uma nova forma de assédio e perseguição?, 16(1), 57-69. Recuperado de: https://doi.org/10.15309/15psd160207

Purdy, N., \& York, L. (2016). A critical investigation of the nature and extent of cyberbullying in two post-primary schools in Northern Ireland. Pastoral Care in Education, 34(1), 13-23. Recuperado de: https://doi.org/10.1080/02643944.2015.1127989

Rice, E., Petering, R., Rhoades, H., Winetrobe, H., Goldbach, J., Plant, A., ... Kordic, T. (2015). Cyberbullying Perpetration and Victimization Among Middle-School Students. American Journal of Public Health, 105(3), e66-e72. Recuperado de: https://doi.org/10.2105/AJPH.2014.302393

Sosa Castilla, C. D., \& Capafóns Bonet, J. I. (2015). Ciberacoso : nuevas formas de acoso infanto-juvenil. Qué hacer cuando la pantalla nos maltrata. Canarias Pediátrica, , 39(2), 105108.

UNICEF (2014). Ocultos a plena luz. Un análisis estadístico de la violencia contra los niños. Recuperado de: https://goo.gl/MNJSk4 
Para referenciar este artículo:

De Barros-Ventura, P., Rodríguez-García, A.M. \& Sola-Reche, J. (2018). Incidencia del ciberbullying en adolescentes de 11 a 17 años en Portugal. EDUTEC, Revista Electrónica de Tecnología Educativa, 64. Recuperado de: http://dx.doi.org/10.21556/edutec.2018.64.1029 